\title{
Uterine Artery Hemodynamic Changes Due to a Cervical Traction Maneuver (Amr's Maneuver) : Randomized Controlled Study
}

\author{
Original Adel M Nada ${ }^{1}$, Emad R Issak², Khaled Kholif', Haitham Torky ${ }^{3}$, Ibrahim Fawzy , \\ Article Reham A. Mohsen
}

Department of Obstetrics and Gynecology, Faculty of Medicine, ${ }^{1}$ Cairo University, ${ }^{3}$ October 6

University, ${ }^{2}$ Contracting Research Organization, Cairo

\begin{abstract}
Aim: This study aimed to measure the impacts of cervical traction on the uterine artery hemodynamics.

Materials and Methods: This study was a randomized-controlled trial. The primary outcome measure was the uterine artery hemodynamics indices: end diastolic velocity (EDV) and systolic/diastolic velocity ratio (S/D ratio).

Results: The study was conducted in one institution in Cairo from January-2017 to March-2017 with 12-month follow up period. Both groups were comparable ( $p$-value $>0.05)$ with regard to the age, BMI, heart rate, blood pressure, gravidity, parity as well as episiotomy. There was no difference $(P>0.05)$ between the two groups regarding vital signs after the intervention. All the patients tolerated the procedure with mild sedation. PSV, EDV, S/D, PI and RI were comparable between both groups before the intervention. However, PSV, S/D ratio, RI and PI were significantly higher $(p$-values $<0.001)$ in the study group than the control group after the intervention. On the other hand, EDV was significantly lower in the study group than the control group after the intervention $(p$-value $<0.001)$. During the follow-up period, no adverse events reported.

Conclusion: Amr's maneuver is effective in changing the uterine artery hemodynamics indices in the direction of decreasing the uterine artery blood flow.
\end{abstract}

Key Words: Amr's Maneuver, cervical traction, end diastolic velocity, peak-systolic velocity, Postpartum hemorrhage, pulsatility index, resistance index, systolic/diastolic velocity ratio

Received: 02 October 2019, Accepted: 04 October 2019

Corresponding Author: Adel M. Nada, Department of Obstetrics and Gynecology, Faculty of Medicine, Cairo University, Cairo Tel.: 0020225218007, E-mail: dr.adel.nada@gmail.com

ISSN: 2090-7265, February 2021, Vol.11, No. 1

\section{INTRODUCTION}

Postpartum hemorrhage (PPH) is the leading cause of maternal mortality worldwide (one-quarter of maternal deaths) with a global prevalence rate ranged from $6-10.8 \%{ }^{[1-3]}$. In Africa and Asia, where most maternal deaths occur, PPH accounts for more than one-third of all maternal mortality ${ }^{[4]}$. Specifically, PPH is the leading cause of maternal mortality in most of the low-income countries. Besides, it is a significant contributor to the severe longterm disability or morbidity encountered in those cases ${ }^{[4-6]}$. In addition, pre-existing anemia can aggravate $\mathrm{PPH}$, in those cases; the loss of a small amount of blood might result in adverse clinical consequences ${ }^{[7]}$.

One of the most important landmarks in the history of discoveries for PPH prevention is the active management of the third stage of labor (AMSTL) which become the gold standard strategy for reducing the PPH incidence ${ }^{[8-10]}$. In the last two decades, many efforts and researches were conducted to improve the preventive measures for $\mathrm{PPH}$ which finalized in 2012 by the addition of misoprostol as an alternative for oxytocin ${ }^{[11]}$. In 2014, Hamdy Amr described his new maneuver by applying the sustained cervical traction for the prevention of $\mathrm{PPH}^{[12]}$. In addition, his maneuver proved effective when added to AMSTL in reduction of the amount of postpartum blood loss ${ }^{[12-15]}$.

Thus, the rationale intended for this current randomized controlled trial was to measure the effects of Amr' Maneuver on the uterine artery hemodynamics indices.

\section{PATIENTS AND METHODS}

Study Design : Pregnant women were enrolled in a single-blinded, controlled, randomized, parallel trial (PACTR Identifier: PACTR201701001984391). The study was conducted in the delivery rooms of Cairo University Hospitals, Egypt during the period from January 2017 to March 2017. The study conformed to the principles of the Declaration of Helsinki and following the Medical Research Involving Human Subjects 
Act. The institutional Bio-Medical Research Ethics Committee approved it (OG4-18-2-17). The purpose of the study was clearly explained in simple and lay Arabic language to all women before their enrollment to the study, and an informed consent form was signed by and obtained from all those enrolled.

Pregnant women meeting all of the following criteria were considered for enrollment: aged 18 years or more, singleton pregnancy and candidate for vaginal delivery at term, and had their delivery in the centers during the period of the study.

Women presenting with any of the following were excluded from the study: ante-partum hemorrhage in this pregnancy, preterm delivery, cases with cervical tears, severe anemia with $\mathrm{HB}<8 \mathrm{mg} / \mathrm{dL}$, and history of pre-existing maternal hemorrhagic conditions such as factor 8 or 9 deficiency or Von Willebrand's disease.

Randomization : For allocation of the participants, a computer-generated list of random numbers was used. Block randomization with a block size of 2 was used with 1:1 ratio of both groups (study and control groups). This was a single blinded study.

Interventions in both groups: For all women in the study, AMSTL (all patients received standard 10 U oxytocin drip ( $30 \mathrm{drops} / \mathrm{min}$ ) was performed. After placental delivery, Amr's maneuver was conducted only for the study group by sustained traction of the anterior and posterior cervical lips by two ovum forceps for duration of 90 seconds.

Procedures : All pregnant women were subjected to detailed history (obstetric, medical and surgical), complete general examination to exclude the presence of any disorders. Obstetrical examinations were made systematically according to each centre's protocol. Only local anesthesia and sedation were used in both groups.

Suitable women who attained vaginal deliveries were closely observed for time of placental delivery. AMSTL was done for all cases.

After the delivery of placenta, transabdominal Doppler US (SONOACE R3, Samsung Inc.) was used to study the uterine artery hemodynamics indices. Then, the maneuver was carried out only for the study group and before the end of the maneuver $(90 \mathrm{sec})$ uterine artery hemodynamics indices was measured for the second time. The same was done for the control group but without doing the maneuver. The uterine artery hemodynamics indices were measured bilaterally and the average of each parameter was recorded in all cases. All measurements were repeated at least three times bilaterally to minimize any sampling error or technical bias.
We used the probe to be perpendicular in the inguinal region beside the uterus (Frequency 3.5 MH). All women were followed-up post-operatively for 6-hours then dismissed and followed-up for 12 months by telephone calls.

Outcome measures : The primary outcome measure was the comparison between uterine artery hemodynamics indices: end diastolic velocity (EDV) and systolic/diastolic velocity ratio (S/D ratio) in both groups before and after the intervention.

The secondary outcome measures were the peaksystolic velocity (PSV), uterine artery resistance index (RI), uterine artery pulsatility index (PI) and the vital signs in both groups before and after the intervention. Also, women discomfort due to the intervention was assessed using a five-point Likert scale ${ }^{[16]}$ where 1 means extremely severe discomfort to the extent the patient cannot tolerate the procedure, 2 severe discomfort but the patient can tolerate the procedure, 3 moderate discomfort, 4 mild discomfort and 5 means no discomfort. Besides, adverse events due to the maneuver were followed-up during a 12-month follow-up period.

Sample size justification : A sample size calculation was estimated to calculate the number of women needed in each group. We made a pilot study upon 20 cases, 10 in each group. We found that EDV was $7.8 \pm 2.8$ in the study group and $10.8 \pm 1.6$ in the control group. We expect that the same figures. Thus, with a significance level $\alpha=0.05$ and a power of $95 \%$ ( $\beta=0.05$ ), equal sample size for both groups, then the required sample size can be determined by 23 women for each arm. Thus, the total sample needed is 46 rounded to 60 subjects to allow for any exclusion.

\section{STATISTICAL ANALYSIS:}

All statistical analyses were made by the intentionto-treat analysis method. All statistical tests were made using a significance level of $95 \%$. P-value $<0.05$ was considered statistically significant. SPSS software (version 20.0, SSPS Inc., Chicago, IL, USA) was used for the statistical analyses. Data were presented as (mean $\pm \mathrm{SD}$ ) or median (interquartile range) for continuous variables and as frequency and percent for categorical variables. Comparisons between groups were made using Chi-square test for categorical variable and the independent t-test or Mann-Whitney test for the continuous variables.

\section{RESULTS}

Ninety-three (93) singleton pregnant women at term who came to the center and were eligible for vaginal delivery during the study period were asked to participate in the study. Eight women declined to participate, 
and 15 women did not meet the inclusion criteria, leaving 70 eligible for randomization with 35 assigned to each group. Ten women were excluded after randomization due to different reasons; 5 from the study group and 5 from the control group. The dispositions of these women are shown in Figure 2.

Baseline characteristics : Both the study and the control groups were comparable with regard their baseline characteristics. There was no statistically significant difference $(P>0.05)$ between the two groups regarding the age, BMI, heart rate, SBP, DBP as well as parity, episiotomy and gravidity, as shown in Tables 1 and 2. Episiotomy was made in 13 (43\%) of women in the study group and in $14(47 \%)$ in the control group, $(P=0.80)$.

Vital signs after the intervention : There was no statistically significant difference $(P>0.05)$ between the two groups regarding the heart rate, SBP and DBP after the intervention.

Patients' discomfort due to the intervention: Women discomfort due to the intervention was assessed using a five-point Likert scale. The mean discomfort score for the study group was $(3.09+0.22)$ and for the control group $(3.22+0.34),(p=0.084)$. All the patients tolerated the procedure with mild sedation. All patients were sedated by $50 \mathrm{mg}$ pethidine IM after fetal delivery.

Uterine artery hemodynamic parameters: Peak-systolic velocity (PSV) was comparable between both groups either before the intervention (p-values 0.25). However, PSV was significantly higher in the study group $(61.8 \pm 15.1)$ than in the control group $(43.2 \pm 5.1)$ after the intervention $(p$-value $<0.001)$.

The end-diastolic velocity (EDV) also was comparable between both groups before the intervention ( $p$-value 0.09). However, EDV was significantly lower in the study group $(5.8 \pm 0.6)$ than in the control group $(14.8 \pm 2.3)$ after the intervention $(p$-value $<0.001)$.

Uterine artery systolic/diastolic velocity ratio (S/D ratio) was comparable between both groups before the intervention ( $p$-value 0.27). However, $\mathrm{S} / \mathrm{D}$ ratio was higher in the study group $(8.2 \pm 1.2)$ than in the control group $(2.9 \pm 0.4)$ after the intervention $(p$-value $<0.001)$.

Uterine artery resistance index (RI) was comparable between both groups before the intervention ( $p$-values 0.13). However, RI was higher in the study group $(0.91 \pm 0.08)$ than in the control group $(0.65 \pm 0.09)$ after doing the intervention $(p$-value $<0.001)$.

Uterine artery pulsatility index (PI) was comparable between both groups before the intervention ( $p$-value 0.23). However, PI was higher in the study group $(1.4 \pm 0.03)$ than in the control group $(1.1 \pm 0.04)$ after doing the intervention $(p$-value $<0.001)$.

Long-term adverse events due to the maneuver: After 12-months follow-up period, no adverse events were reported in the study group. 

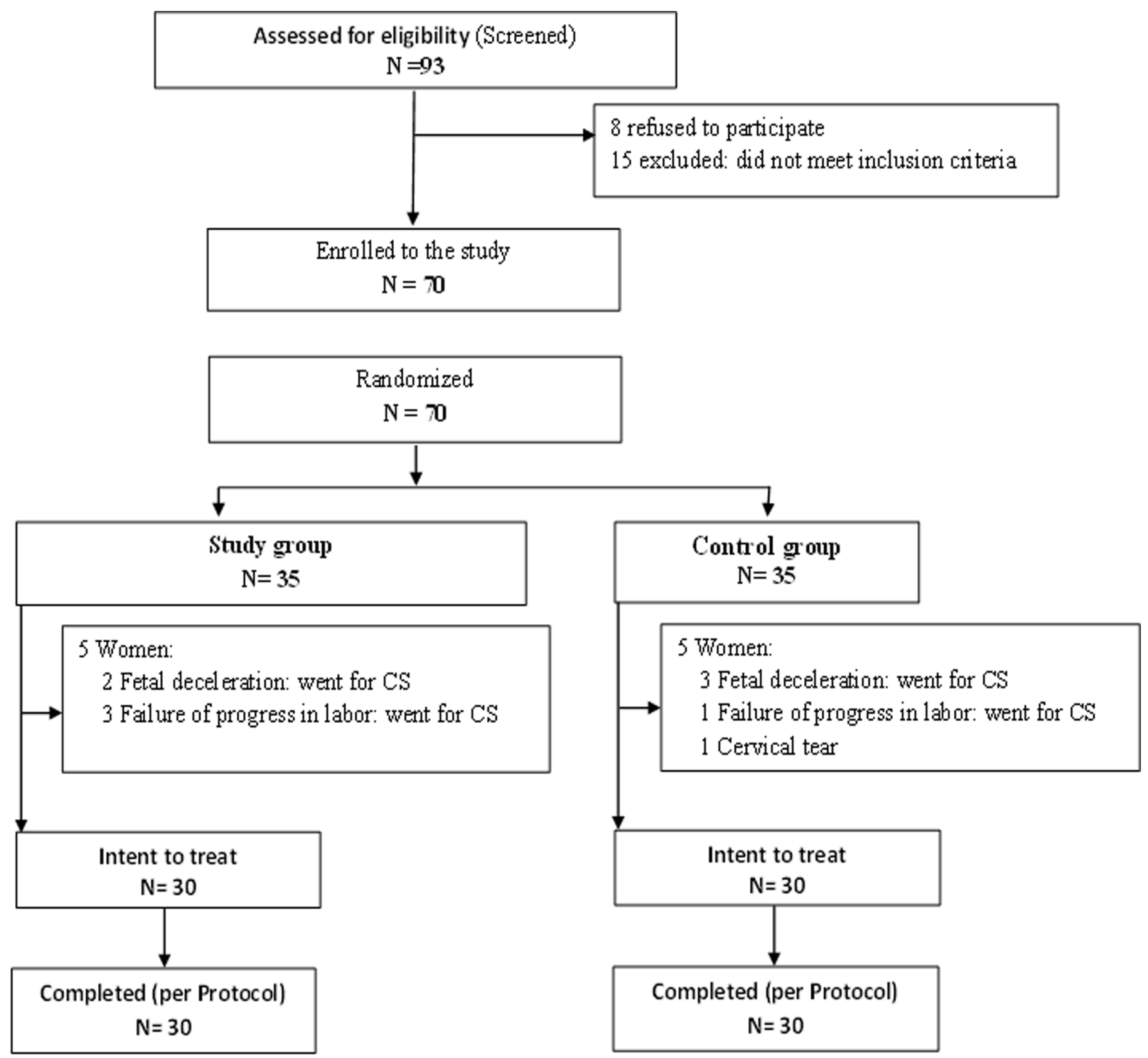

Fig.1: CONSORT diagram 


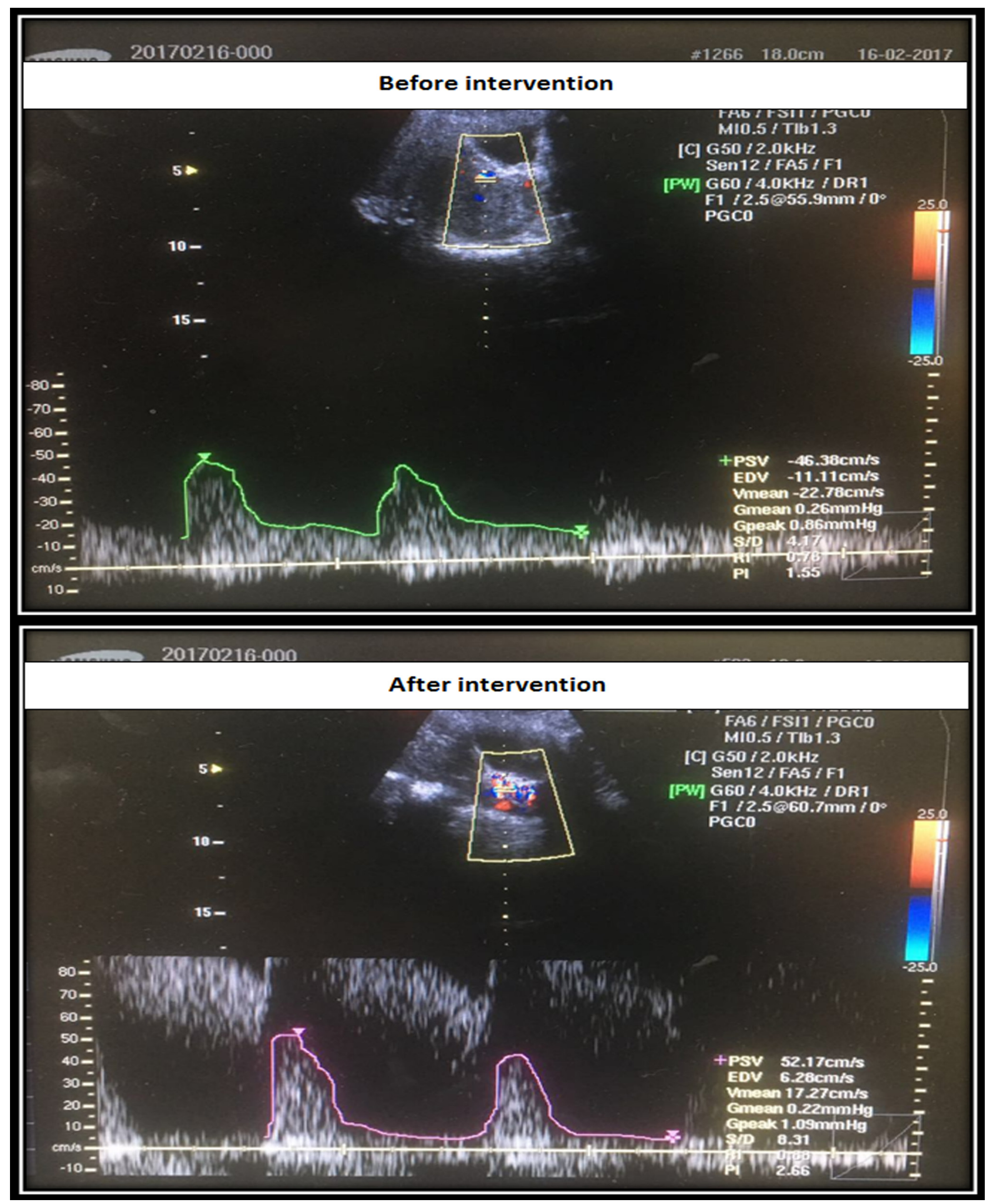

Fig. 2: Changes in hemodynamic indices due to Amr's maneuver 
Table 1: Patients' Characteristics

\begin{tabular}{|c|c|c|c|}
\hline & $\begin{array}{l}\text { Study group } \\
\quad \mathrm{N}=30\end{array}$ & $\begin{array}{c}\text { Control group } \\
\mathrm{N}=30\end{array}$ & $P$-value \\
\hline Age in years, mean (SD) & $28.8(4.9)$ & $29.1(4.5)$ & 0.78 \\
\hline BMI (kg/m2), mean (SD) & $27.8(3.3)$ & $27.9(3.5)$ & 0.88 \\
\hline Parity, mean (SD) & $1.8(1.4)$ & $1.6(1.3)$ & 0.57 \\
\hline Discomfort, mean (SD) & $3.09(0.22)$ & $3.22(0.34)$ & 0.084 \\
\hline Episiotomy, n (\%) & $13(43 \%)$ & $14(47 \%)$ & 0.80 \\
\hline Primigravida, n (\%) & $7(23 \%)$ & $6(20 \%)$ & 0.75 \\
\hline
\end{tabular}

Table 2: Vital signs and uterine artery hemodynamic parameters

\begin{tabular}{|c|c|c|c|c|c|c|}
\hline & \multicolumn{3}{|c|}{ Before intervention } & \multicolumn{3}{|c|}{ After intervention } \\
\hline & Study & Control & $p$ & Study & Control & $p$ \\
\hline \multicolumn{7}{|l|}{ Vital signs } \\
\hline Heart rate & $82.8(6)$ & $81.1(7.5)$ & 0.53 & $78.2(6.6)$ & $76.2(5)$ & 0.35 \\
\hline SBP & $120(8.3)$ & $118(9.6)$ & 0.44 & $119.7(8.1)$ & $118.3(10.2)$ & 0.57 \\
\hline DBP & $76.8(5.6)$ & $74(6.7)$ & 0.08 & $77.2(5.5)$ & $74.5(7)$ & 0.12 \\
\hline \multicolumn{7}{|c|}{ Uterine artery hemodynamic parameters } \\
\hline $\begin{array}{l}\text { Peak-systolic } \\
\text { velocity (PSV) }\end{array}$ & $40.9(9)$ & $43.3(6.7)$ & 0.25 & $61.8(15.1)$ & $43.2(5.1)$ & $P<0.001$ \\
\hline $\begin{array}{l}\text { End-diastolic } \\
\text { velocity (EDV) }\end{array}$ & $15.6(3.4)$ & $14.4(2)$ & 0.09 & $5.8(0.6)$ & $14.8(2.3)$ & $P<0.001$ \\
\hline $\begin{array}{l}\text { Uterine artery } \\
\text { systolic/diastolic } \\
\text { velocity ratio } \\
\text { (S/D ratio) }\end{array}$ & $2.6(0.5)$ & $2.8(0.6)$ & 0.17 & $8.2(1.2)$ & $2.9(0.4)$ & $P<0.001$ \\
\hline $\begin{array}{l}\text { Uterine artery } \\
\text { resistance } \\
\text { index }(\mathrm{RI})\end{array}$ & $0.62(0.02)$ & $0.63(0.3)$ & 0.13 & $0.91(0.08)$ & $0.65(0.09)$ & $P<0.001$ \\
\hline $\begin{array}{l}\text { Uterine artery } \\
\text { pulsatility } \\
\text { index }(\mathrm{PI})\end{array}$ & $1.01(0.04)$ & $1.0(0.02)$ & 0.23 & $1.4(0.03)$ & $1.1(0.04)$ & $P<0.001$ \\
\hline
\end{tabular}




\section{DISCUSSION}

This randomized controlled study was conducted to understand the mechanism behind Amr's maneuver through exploring the uterine artery hemodynamic indices with or without the maneuver in question.

The results of this current study showed that the vital signs; heart rate and blood pressure were comparable between both groups before and after doing the maneuver. In addition, PSV, EDV, S/D, PI \& RI were comparable between both groups before the intervention. However, PSV, S/D ratio, RI \& PI were higher in the study group than the control group after the intervention. On the other hand, EDV was significantly lower in the study group than the control group after the intervention. These aforementioned changes in the uterine artery hemodynamic indices resulted from the intervention can explain the reduction of blood losses when using the maneuver as reflected in a reduction in the uterine artery blood flow.

One explanation to these uterine artery hemodynamic changes is a kinking of the redundant uterine arteries due to the maneuver results in slow blood flow that allows more suitable condition for clotting and thrombin formation ${ }^{[17,18]}$. Another explanation can be that the reflex contraction of the uterine musculature due to the maneuver.

To the best of our knowledge, this is the first study that investigates the uterine artery blood flow after delivery. The evidence of the current study is strong enough to prove the efficacy of the maneuver in changing the uterine artery hemodynamics favoring the reduction of uterine blood flow in the uterine arteries; thus, reducing the loss of blood.

\section{CONCLUSION}

Amr's Maneuver when added to AMSTL is effective in decreasing the blood flow in the uterine arteries; thus, reducing the amount of postpartum blood loss.

\section{ETHICAL APPROVAL}

All procedures performed in studies involving human participants were in accordance with the ethical standards of the institutional and/or national research bioethical committee and with the 1964 Helsinki declaration and its later amendments or comparable ethical standards.

\section{CONFLICT OF INTEREST}

There are no conflicts of interests.

\section{REFERENCES}

1. Fawole B, Awolude OA, Adeniji AO, et al. WHO recommendations for the prevention of postpartum haemorrhage: RHL guideline. The WHO Reproductive Health Library. Geneva: World Health Organization. Revised 1 May 2010.

2. Calvert C, Thomas SL, Ronsmans C, Wagner KS, Adler AJ, et al. Identifying Regional Variation in the Prevalence of Postpartum Haemorrhage: A Systematic Review and Meta-Analysis. PLoS ONE 2012; 7(7): e41114. doi:10.1371/journal. pone. 0041114

3. Ndola Prata, Suzanne Bell, and Karen Weidert. Prevention of postpartum hemorrhage in lowresource settings: current perspectives. Int J Womens Health. 2013; 5: 737-752.

4. Khan KS, Wojdyla D, Say L, Gulmezoglu AM, Van Look PFA. WHO analysis of causes of maternal death: a systematic review. The Lancet 2006; 367:1066-1074.

5. Campbell OM, Graham WJ. Lancet Maternal Survival Series Steering Group. Strategies for reducing maternal mortality: getting on with what works. Lancet. 2006; 368 (9543): 1284-99.

6. World Health Organization. World Health Organization multicountry survey on maternal and newborn health. Geneva: WHO; 2012

7. World Health Organization. Managing complication in pregnancy and childbirth: a guide for midwives and doctors. Geneva: WHO; 2000. Available from: http://www.who. int/reproductivehealth/publications/maternal_ perinatal_health/9241545879/en/index.html

8. SPENCER PM. Controlled cord traction in management of the third stage of labour.Br Med J. 1962 Jun 23; 1(5294):1728-32.

9. International Confederation of Midwives (ICM) International Federation of Gynaecology and 
Obstetrics (FIGO) Joint Statement: Management of the Third Stage of Labour to Prevent Postpartum Haemorrhage. New York, NY: FIGO and ICM; 2003.

10. Begley CM, Gyte GM, Devane D, McGuire W, Weeks A. Active versus expectant management for women in the third stage of labour. Cochrane DatabaseSyst Rev.2011(11). Available from: http:// onlinelibrary.wiley.com/doi/10.100214651858/. CD007412.pub3/abstract

11. Dept of Reproductive Health and research, World Health Organization (WHO) WHO Recommendations for the Prevention and Treatment of Postpartum Haemorrhage. Geneva: WHO; 2012.

12. Hamdy, AMR. A new maneuver for the prevention of postpartum hemorrhage. The Journal of Obstetrics and Gynecology of India, 2014; 65(4):241.

13. T. Anitha Catherine Preethima, V. Jayashree and K. Latha. A study of sustained cervical traction in the prevention of postpartum hemorrhage. Int. J. Modn. Res. Revs. 2016; 4 (11): 1368-1371
14. Subramaniyam C, Chandran S, Priya S Comparative Study on Prevention of Postpartum Hemorrhage by Routine Active Management of Third Stage of Labor Versus Active Management of Third Stage of Labor with AMR'S Maneuver in Madurai Medical College, Tamil Nadu, India. Int J Sci Stud 2017; 5(1):7073-.

15. Hamdy A, Azmy O, Lotfy R, Attia AA, Elsherbini MM, Al Sawaf A, Soliman MM, Sharaf MF, Kamel A, Abd El-Raouf MN, Salem S, Rasheed MA, Torky H, Issak ER. Multicenter randomized controlled trial assessing the impact of a cervical traction maneuver (Amr's maneuver) on the incidence of postpartum hemorrhage. Int J Gynaecol Obstet. 2019 Jan;144(1):5661-.

16. Likert R. A technique for measurement of attitudes. Arch Psychol 1932;140:155-.

17. Chibbar R, Miller F, Mitchell F. Synthesis of Oxytocin in amnion, chorion and decidua may influence the timing of human parturition. J Clin Invest. 1993;91:185-92. doi:10.1172/JCI116169.

18. Abdel-Sater KA. Physiological positive feedback mechanisms. Am J Biomed Sci. 2011; 3(2):145-55. doi:10.5099/aj110200145. 